\title{
Electrogyration effect in lead germanate crystal family.
}

\section{Electrogyration in the solid solutions based on lead germanate crystals}

\author{
Shopa Y. ${ }^{1,2}$, Adamenko D. ${ }^{2}$, Vlokh R. ${ }^{2}$ and Vlokh O. ${ }^{2}$ \\ ${ }^{1}$ Ivan Franko National University of Lviv, 8 Kyrylo and Mefodiy St., 79005 \\ Lviv, Ukraine, e-mail: shopa@physics.wups.lviv.ua \\ ${ }^{2}$ Institute of Physical Optics, 23 Dragomanov St., 79005 Lviv, Ukraine, \\ e-mail:vlokh@ifo.lviv.ua
}

Received: 01.10.2007

\begin{abstract}
We present the results of studies of electrogyration effect in the solid solutions $\mathrm{Pb}_{5}\left(\mathrm{Ge}_{(1-\mathrm{x})} \mathrm{Si}_{\mathrm{x}}\right)_{3} \mathrm{O}_{11}$ and $\left(\mathrm{Pb}_{(1-\mathrm{x})} \mathrm{Ba}_{\mathrm{x}}\right)_{5} \mathrm{Ge}_{3} \mathrm{O}_{11}$. We show that substitutions of $\mathrm{Pb}$ with $\mathrm{Ba}$ and $\mathrm{Ge}$ with $\mathrm{Si}$ do not lead to the changes in electrogyration coefficients, defined in the units of reciprocal electric polarization. At the same time, the main contribution to the changes in electrogyration coefficients, defined in the units of reciprocal electric field strength, is given by critical behaviour of the dielectric permittivity in the vicinity of Curie temperature. It follows from a simple thermodynamic analysis that a hypothetical tricritical point can exist on the $x, T$-phase diagram for the solid $\left(\mathrm{Pb}_{(1-\mathrm{x})} \mathrm{Ba}_{\mathrm{x}}\right)_{5} \mathrm{Ge}_{3} \mathrm{O}_{11}$ solutions.
\end{abstract}

Keywords: electrogyration, ferroelectric phase transition, optical activity, lead germanate crystals, phase diagram

PACS: $33.55 . A d, 78.20 . J q, 78.20 . E k, 77.80 .-\mathrm{e}$

\section{Introduction}

Lead germanate crystals $\left(\mathrm{Pb}_{5} \mathrm{Ge}_{3} \mathrm{O}_{11}\right.$, abbreviated often as PGO) are proper ferroelectrics exhibiting a phase transition at the temperature $T_{c}=450 \mathrm{~K}$ [1]. The crystals belong to the hexagonal point symmetry group $\overline{6}$ above the Curie temperature, whereas the inversion symmetry axis disappears below this point and the symmetry is reduced to the point group 3 . Ferroelectric properties of the lead germanate crystals has been revealed more than 30 years ago. Though a number of the known polar dielectrics exceed one million [2], PGO crystals remain, probably, a unique example of materials where the mentioned symmetry change occurs at the phase transition. From the viewpoint of studies of optical activity phenomenon, such the change of symmetry at the phase transition is very convenient. Then the optical rotation in both the paraelectric and ferroelectric phases

Ukr. J. Phys. Opt. 2007, V8, №4 
could be directly measured for the light propagating along the optic axis, without the accompanying linear optical birefringence, while the application of external electric field along the optic axis induces no linear birefringence in this direction. Temperature behaviour of the optical activity in the proper ferroelectrics should reflect behaviour of the order parameter, i.e. the spontaneous electric polarization [3]. Therefore the electrogyration induced by a biasing field shows the same temperature behaviour as the dielectric permittivity. Thus, the studies of the temperature changes of natural optical activity and electrogyration would allow one to reveal character of the phase transition, change of the point symmetry group at the phase transition, etc.

In case of the PGO crystals, the spontaneous polarization as the order parameter is linearly coupled with the optical activity, which appears below $T_{c}$. This fact has been revealed for the first time by $\mathrm{H}$. Iwasaki et al. [1]. The main contribution to the spontaneous polarization originates from shifting of $\mathrm{Pb}^{2+}$ ions along $c$ axis [4], while the optical activity and the change of its sign under domain switching is caused by the rotation of $\mathrm{GeO}_{4}^{4-}$ tetrahedrons [4]. In other words, while the $\mathrm{Pb}^{2+}$ ions move out of the tetrahedron prism, the oxygen ions also undergo a shift inducing twisting of the $\mathrm{GeO}_{4}^{4-}$ tetrahedrons [4]. The lead germanate crystals and solid solutions on their basis could serve as model objects when analyzing mechanisms of the natural optical activity (i.e., the electrogyration induced by spontaneous polarization) and the electrogyration effect. In particular, this is due to simplicity of chemical elements substitutions or doping. There has been a lot of experimental results concerned with the electrogyration effect in the lead germanate family crystals. For instance, we may refer a reader to the results of studies of $\mathrm{Pb}_{5}\left(\mathrm{Ge}_{(1-\mathrm{x})} \mathrm{Si}_{\mathrm{x}}\right)_{3} \mathrm{O}_{11}$ and $\left(\mathrm{Pb}_{(1-\mathrm{x})} \mathrm{Ba}_{\mathrm{x}}\right)_{5} \mathrm{Ge}_{3} \mathrm{O}_{11}$ solid solutions [1, 5-8].

Searching for the solid solutions (or doped crystals) with high values of electrogyration rotation is still interesting from the viewpoint of applications of new electrogyration materials for operating optical radiation. Let us note here that, besides of chemical substitution, doping of pure crystals with some chemical elements could be also suspected as promising for this aim. For example, the electrogyration effect has been studied for the PGO crystals doped with such the impurities as Eu [9], Cd and Nd [10]. Doping of the PGO crystals with different chemical elements can lead to shifts in the phase transition temperature. It is useful for applications in the optical storage, lasing and fabrication of thin ferroelectric film memory units. The photorefractive effect has been studied for the PGO crystals doped with $\mathrm{Cu}$ and $\mathrm{Nd}[11,12]$. Concerning electrogyration effect in the doped PGO crystals, one can remind that the magnitude of electrogyration coefficient in $\mathrm{Pb}_{5} \mathrm{Ge}_{3} \mathrm{O}_{11}: \mathrm{Cr}$ at $T_{c}$ is larger than that observed for the pure crystals [13]. Thus, the present paper is devoted to analysis of electrogyration effect in the lead germanate family of crystals, with the emphasis on the influence of chemical substitution on its magnitude. 


\section{Electrogyration in the solid solutions $\mathrm{Pb}_{5}\left(\mathrm{Ge}_{(1-\mathrm{x})} \mathrm{Si}_{\mathrm{x}}\right)_{3} \mathrm{O}_{11}$ (PGSO)}

The solid solutions of lead germanate-silicate $\left(\mathrm{Pb}_{5}\left(\mathrm{Ge}_{(1-\mathrm{x})} \mathrm{Si}_{\mathrm{x}}\right)_{3} \mathrm{O}_{11}\right.$ or simply PGSO) crystals turn out to be very suitable for the studies of electrogyration effect in the course of phase transition. Increasing $\mathrm{Si}$ concentration leads to a drastic decrease in the Curie temperature (e.g., it is about $313 \mathrm{~K}$ for $x=0.40$, while for the pure PGO crystals we have the Curie temperature $450 \mathrm{~K}$ ). In addition, the conductivity in these solid solutions decreases with decreasing temperature [14]. The Curie temperature for the crystals substituted with $\mathrm{Ba}$ as well as $\mathrm{Si}$ also decreases $[5-8,15]$, thus permitting to apply higher electric fields without appearance of noticeable electric currents. The measurements of optical activity in the PGSO crystals have been carried out on single-domain samples with the silicon concentrations $x=0,0.03,0.05,0.10,0.20$ and 0.40 . No biasing electric field has been applied, while the electrogyration effect has been studied in the conditions of zero-frequency biasing field applied along $\mathrm{Z}$ axis.

Substitution of Ge ions with Si ones leads to decrease in the specific rotatory power at the same temperatures, though this can be easily explained by the corresponding Curie
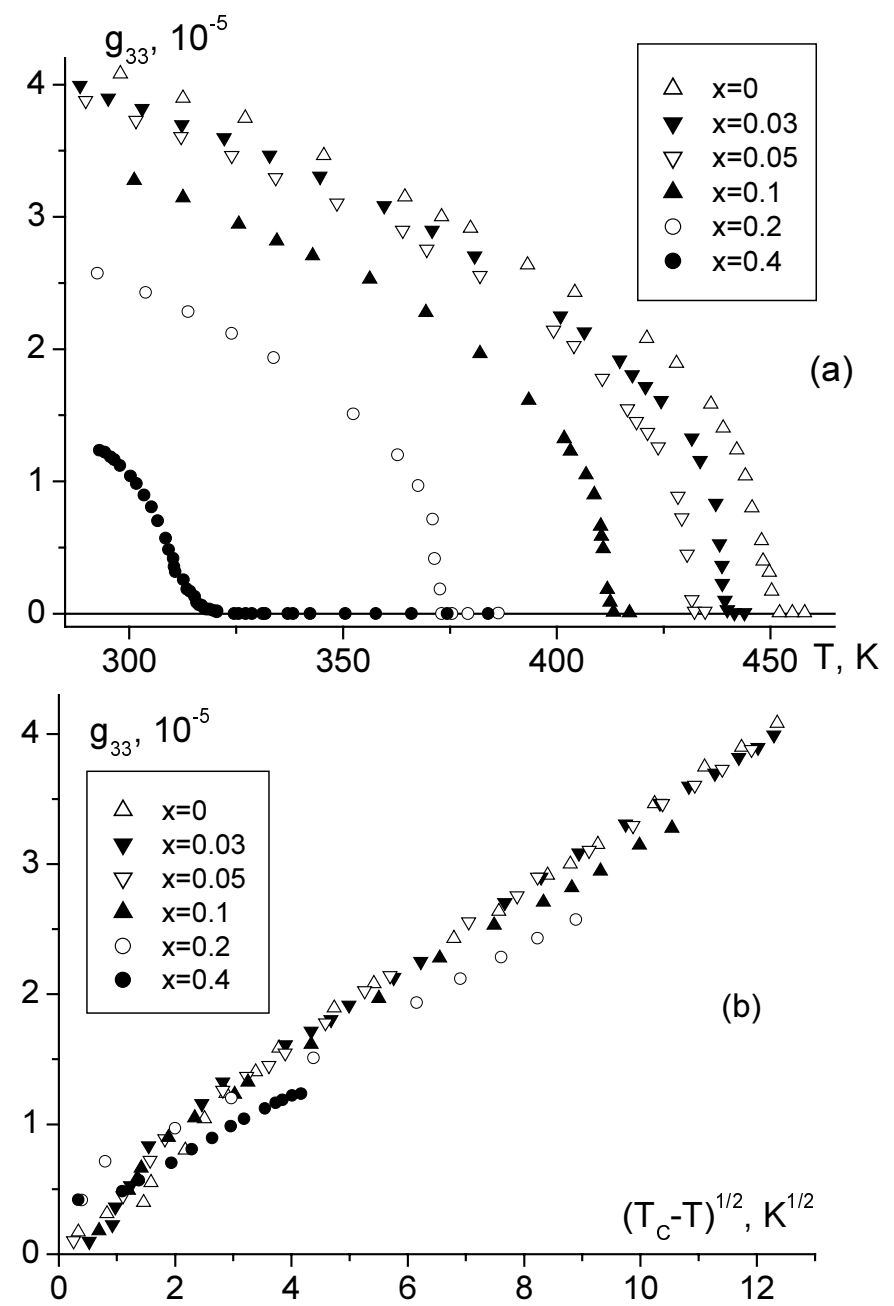

Fig. 1. Dependences of the gyration tensor component $g_{33}$ for the PGSO solid solutions on the temperature (a) and on the parameter $\left(T_{c}-T\right)^{1 / 2}(\mathrm{~b})$. 
temperature decrease (Fig. 1). The rate of the phase transition temperature decrease with increasing Si concentration is linear. In Fig. 1a the temperature dependences of optical activity are presented in the units of gyration tensor component $g_{33}=\rho \lambda n_{o} / \pi$ (with $\rho$ being the specific angle of polarization plane rotation, $\lambda$ the wavelength of optical radiation and $n_{o}$ the ordinary refractive index). When calculating temperature dependences of the gyration tensor component for the solid solutions of PGSO, we have taken the temperature dependence of the refractive index into account. At the same time, the value of the refractive index for the PBaGO solid solutions has been chosen as $n_{o}=2.12 \quad[16]$.

As one can see from Fig. 1b, the dependence of the gyration tensor component $g_{33}$ on $\left(T_{c}-T\right)^{1 / 2}$ is almost linear. This corresponds to classical temperature behaviour of the order parameter in the course of second-order phase transitions.

As seen from Fig. 2, the substitution of Ge with Si hardly affects the optical rotation power (the corresponding data for the temperature dependence of spontaneous polarization are taken from [15]). It is also worthwhile that the dependences presented in Fig. 2 for PGSO crystals can be better approximated with a higher-order polynomial:

$$
g_{33}=\tilde{\gamma}_{333} P_{3}^{s}+\tilde{\beta}_{3333} P_{3}^{s} P_{3}^{s}+\tilde{\delta}_{33333} P_{3}^{s} P_{3}^{s} P_{3}^{s},
$$

where $P_{3}^{s}$ is the spontaneous polarization, $\tilde{\gamma}_{333}, \tilde{\beta}_{3333}$ and $\tilde{\delta}_{33333}$ are respectively the coefficients of linear, quadratic and cubic electrogyration represented in the units of reciprocal polarization.

Since the quadratic term in Eq. (1) is forbidden by the symmetry conditions, one can pick out a contribution of cubic electrogyration induced by spontaneous polarization. Then Eq. (1) may be rewritten as

$$
g_{33}=\tilde{\gamma}_{333} P_{3}^{s}+\tilde{\delta}_{33333} P_{3}^{s} P_{3}^{s} P_{3}^{s},
$$

where $\tilde{\delta}_{33333}$ is the component of fifth-rank axial tensor. The coefficient of linear

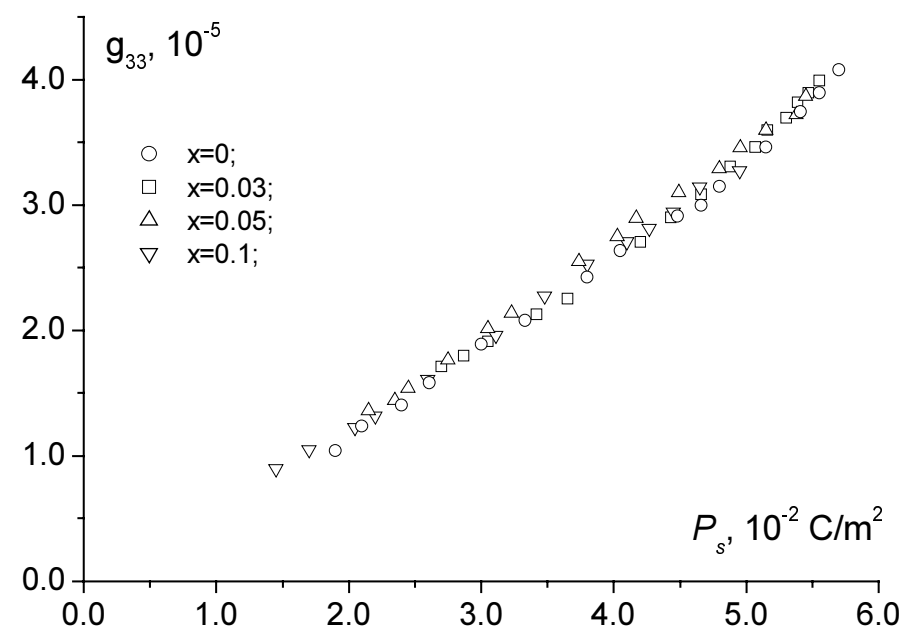

Fig. 2. Dependences of the gyration tensor component $g_{33}$ on the spontaneous polarization for the PGSO solid solutions. 


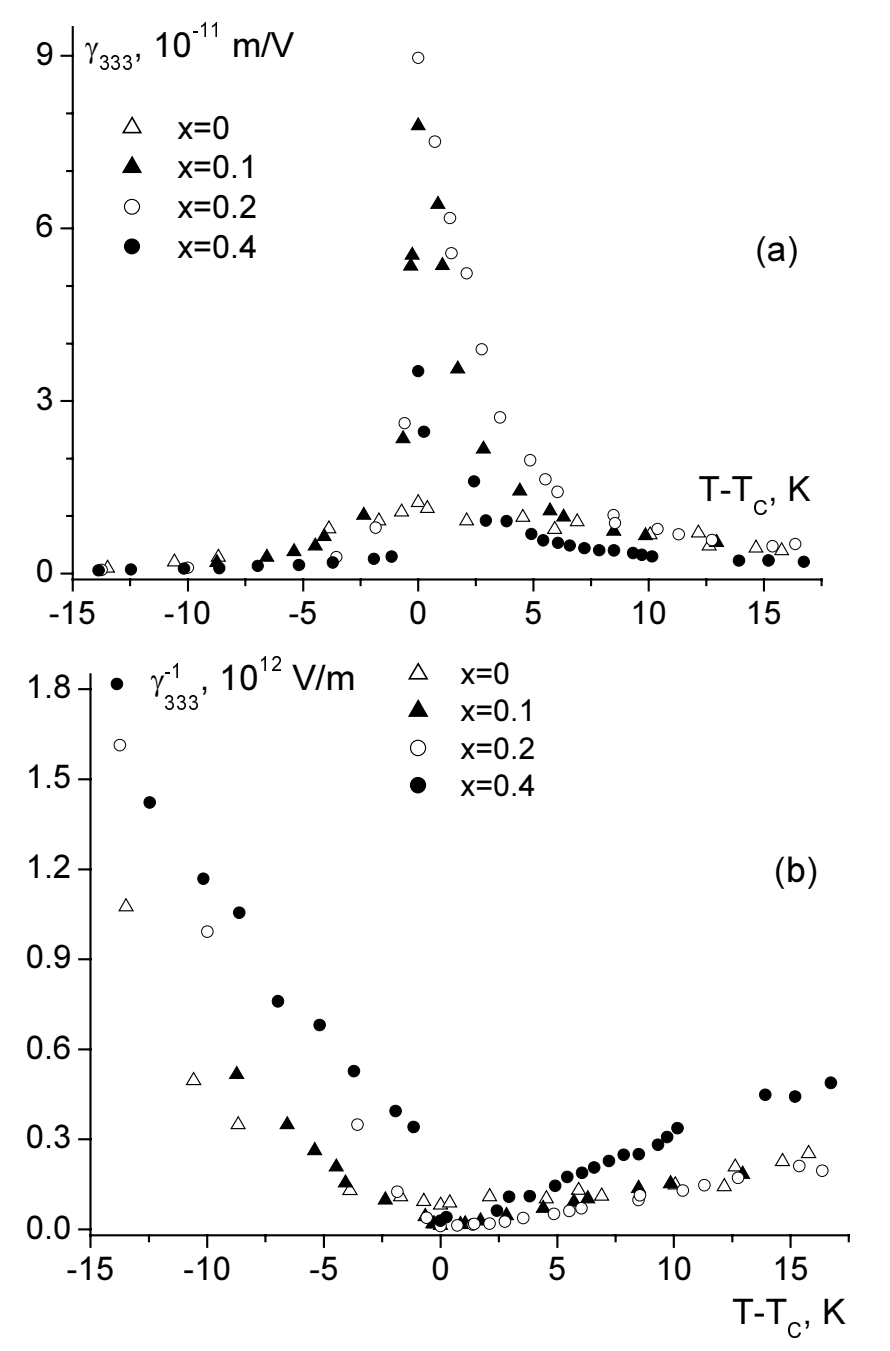

Fig. 3. Temperature dependences of the linear electrogyration coefficient $\gamma_{333}$ (a) and it reciprocal value $\gamma_{333}^{-1}$ (b) for the PGSO solid solutions.

electrogyration $\tilde{\gamma}_{333}$ calculated for PGSO crystals at $x=0-0.10$ is equal to $(59.7 \pm 0.6) \times 10^{-5} \mathrm{~m}^{2} / \mathrm{C}$, whereas the cubic electrogyration parameter is equal to $\tilde{\delta}_{33333}=(3.5 \pm 0.2) \times 10^{-2} \mathrm{~m}^{6} / \mathrm{C}^{3}$.

The temperature dependences of the linear electrogyration obtained by us (see Fig. 3) are in general similar to those for the dielectric permittivity [15]. It is seen from Fig. $3 \mathrm{~b}$ that the temperature dependence of $\gamma_{333}^{-1}$ parameter almost satisfies the known Curie-Weis law.

The field dependences of the optical activity increment measured in the paraelectric phase become nonlinear in the vicinity of $T_{c}$ (Fig. 4). This is readily explained by a fieldinduced shift of phase transition temperature towards higher temperatures. As a consequence, we have used the slopes of these dependences at $E_{z}=0$ in order to determine correctly the electrogyration coefficients. In the paraelectric phase, we have observed the so-called double hysteresis loops in the vicinity of $T_{c}$ (see Fig. 5). They 

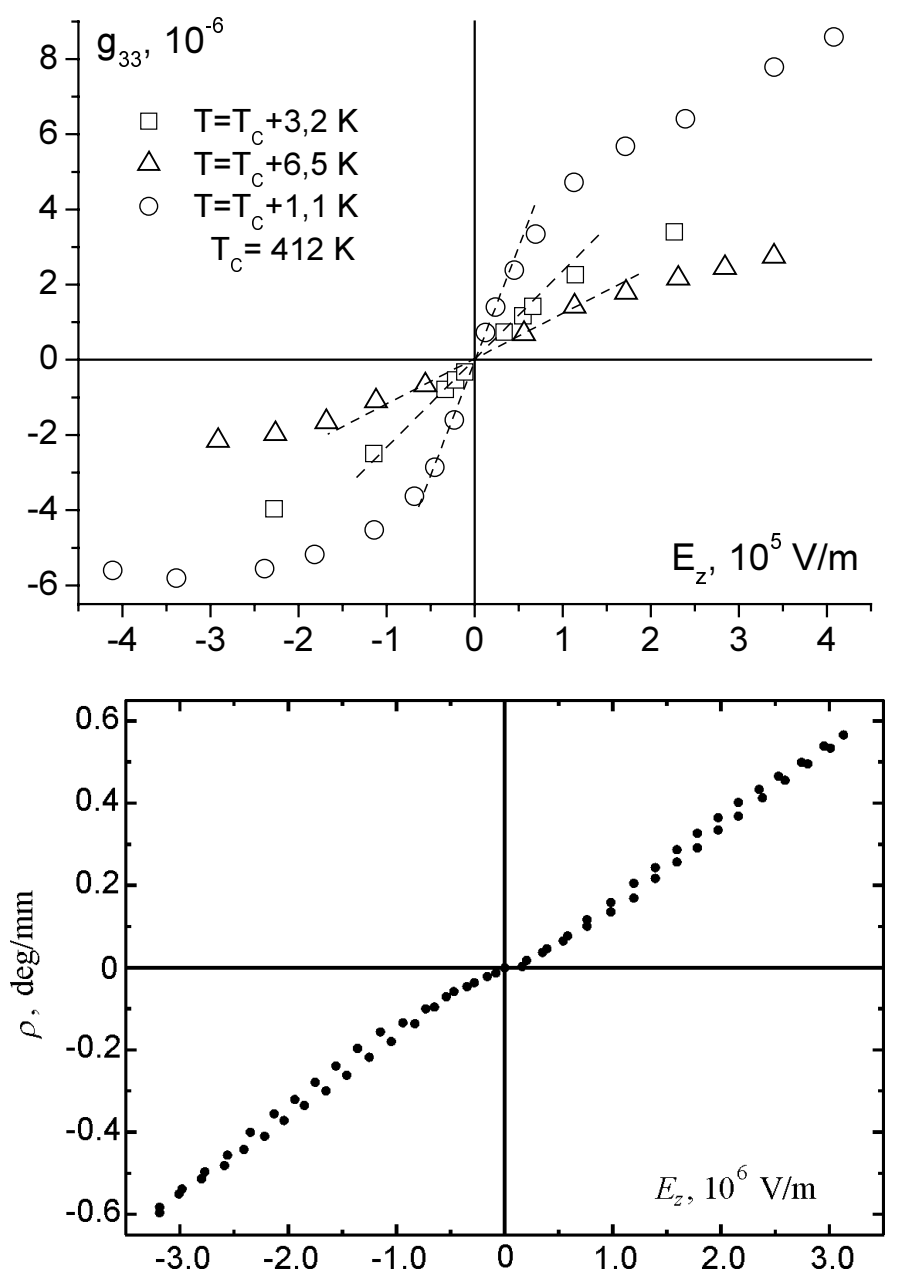

Fig. 4. Dependences of the gyration tensor component $g_{33}$ for the PGSO solid solution $(x=0.1)$ measured in the vicinity of $T_{c}$. The dashed lines are slopes taken at $E_{z}=0$.
Fig. 5. Double hysteresis loop of the induced optical activity for the PGSO crystals $\quad(x=0.4)$ measured at $T=327.1 \mathrm{~K}$.

should be a result of shift of the phase transition towards higher temperatures and production of ferroelectric domains by the electric field.

\section{Electrogyration in the solid solutions $\left(\mathrm{Pb}_{(1-\mathrm{x})} \mathrm{Ba}_{\mathrm{x}}\right)_{5} \mathrm{Ge}_{3} \mathrm{O}_{11}$ (PBaGO)}

In general, the solid solutions of $\left(\mathrm{Pb}_{(1-\mathrm{x})} \mathrm{Ba}_{\mathrm{x}}\right)_{5} \mathrm{Ge}_{3} \mathrm{O}_{11}$ (abbreviated as $\mathrm{PBaGO}$ ) manifest the same type of phase transition as that in the pure PGO crystals. The temperature dependences of optical activity for the solid solutions characterized with the concentrations $x=0,0.02$ and 0.05 are presented in Fig. 6a. It is seen that the substitution of $\mathrm{Pb}$ with $\mathrm{Ba}$ yields essential decrease in the phase transition temperature (from $450 \mathrm{~K}$ for the pure PGO to approximately $356.4 \mathrm{~K}$ for the PBaGO with $x=0.05$ ). The second result is increased range of smearing of the phase transition. Still the most interesting is the fact that the temperature dependence of the optical activity changes its behaviour in case of the PbaGO solid solutions. For example, the phase transition in the pure PGO crystals is of the second order, while the temperature dependences of optical activity (i.e. the order parameter) in the PBaGO solid solutions are far from the classical behaviour usual for second-order phase transitions. This is testified by definitely non- 


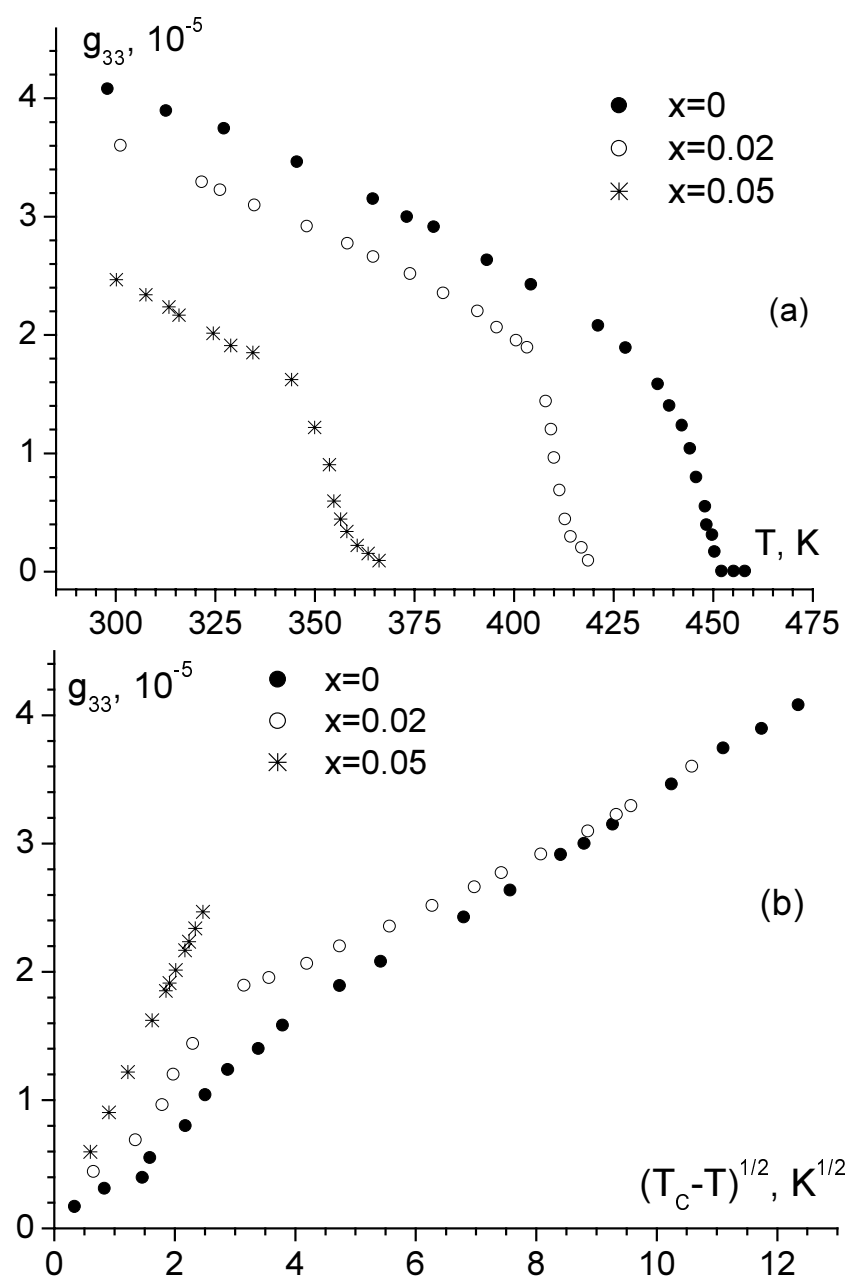

Fig. 6. Dependences of optical activity in the PBaGO solid solutions on the temperature (a) and the parameter $\left(T_{c}-T\right)^{1 / 2}(\mathrm{~b})$.

linear behaviour of the parameter $g_{33}$ on $\left(T_{c}-T\right)^{1 / 2}$ (see Fig. 6b). One can assume that a tricritical point (i.e., a change of the order of phase transition from the second to the first one) exists on the $x, T$-phase diagram of the PBaGO somewhere at $x>0.05$.

Fig. 7a shows that the peak electrogyration value achieved at $T_{c}$ in the solid solutions with $x=0.02$ is higher, when compare with the corresponding value measured for the pure PGO crystals. Nonetheless, the magnitude of the anomaly observed for the electrogyration coefficient falls rapidly down already for the concentration $x=0.05$. This may be caused by smearing of the phase transition occurred in the compounds characterized with the concentration of Ba equal to $x=0.05$. The temperature behaviour of the reciprocal electrogyration coefficient almost satisfies the Curie-Weiss law (see Fig. 7b).

\section{Discussion}

The $x, T$-phase diagrams for the solid solutions under test are presented in Fig. 8. In both cases the substitution of lead and germanium ions leads to decrease of $T_{c}$, though the 

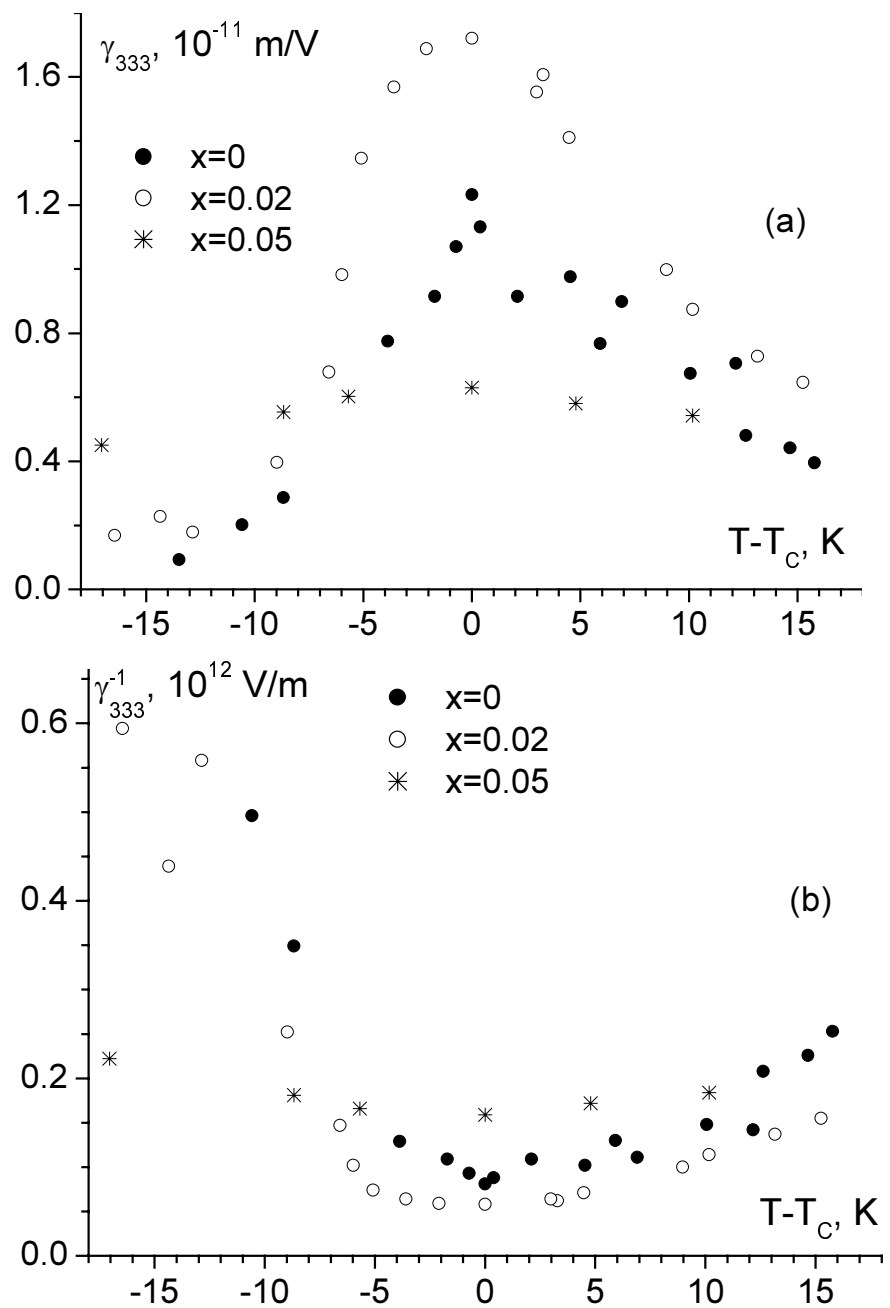

Fig. 7. Temperature dependences of the electro-gyration coefficient (a) and its reciprocal value (b) for the PBaGO solid solutions.

substitution of lead with barium induces more essential change in the phase transition temperature. This result correlates with the conclusion [4] that the main contribution to the spontaneous polarization comes from shifting of $\mathrm{Pb}^{2+}$ ions.

Let us remind some basic relations for the proper second-order ferrolelectric phase transitions (see, e.g., [17]). Let us write the thermodynamic potential as

$$
F=F_{0}+\alpha\left(T-T_{c}\right)^{(s)} P^{2}+\frac{1}{2} \beta^{(s)} P^{4}+\frac{1}{3} \delta^{(s)} P^{6}+\ldots .
$$

Then the following relations for the spontaneous polarization and the gyration tensor component are straightforward:

$$
{ }^{(s)} P^{2}=-\frac{8 \pi \alpha\left(T-T_{c}\right)}{\beta}
$$

and

$$
g_{33}= \pm \frac{2 \tilde{\gamma}_{333}}{\pi}\left[\frac{2 \pi \alpha}{\beta}\left(T_{c}-T\right)\right]^{1 / 2}
$$




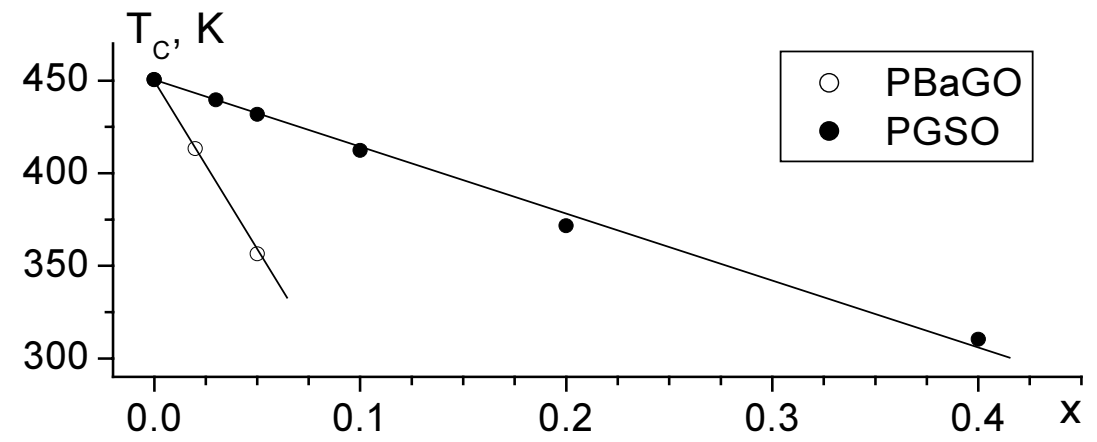

Fig. 8. $x, T$-phase diagram for the PGSO and PBaGO solid solutions.

The temperature dependences of the dielectric permittivity and the electrogyration coefficient are defined respectively by the relations

$$
\varepsilon_{33}=\frac{C}{T-T_{c}} \text { at } T>T_{c}
$$

and

$$
\varepsilon_{33}=\frac{C}{2\left(T_{c}-T\right)} \text { at } T<T_{c} \text {, }
$$

and

$$
\begin{aligned}
& \gamma_{333}=\frac{C \varepsilon_{0} \tilde{\gamma}_{333}}{T-T_{c}} \text { for } T>T_{c}, \\
& \gamma_{333}=\frac{C \varepsilon_{0} \tilde{\gamma}_{333}}{2\left(T_{c}-T\right)} \text { for } T<T_{c},
\end{aligned}
$$

where $C=(2 \alpha)^{-1}$ is the Curie-Weiss constant and $\gamma_{33}=\tilde{\gamma}_{333} \varepsilon_{0}\left(\varepsilon_{33}-1\right) \simeq \tilde{\gamma}_{333} \varepsilon_{0} \varepsilon_{33}$ (with $\varepsilon_{0}$ being the permittivity of free space). Hence, we arrive at the Curie-Weiss law for the electrogyration coefficients:

$$
\frac{\left(\gamma_{333}^{-1}\right)_{T<T_{c}}}{\left(\gamma_{333}^{-1}\right)_{T>T_{c}}}=2 .
$$

It follows from Tables 1 and 2 that the Curie-Weiss law is approximately satisfied for the PGSO and PBaGO solid solutions (the temperature dependences of the dielectric permittivity and spontaneous polarization have been taken from $[8,15])$. It is interesting to notice that the Curie constant increases with increasing amount of the substitution ions. At the same time, the coefficients of thermodynamic potential $\alpha$ and $\beta$ then decrease. The value of the coefficient $\alpha$ obtained by us from the present optical measurements is in good agreement with the corresponding value obtained using the dielectric measurements $[1,7,13]$. 
Notice also that the two coefficients of thermodynamic potential mentioned above tend to zero with increasing substitution ion concentration and, quite probably, can change their signs at further increasing of $x$. Such behaviour of thermodynamic coefficients is peculiar for a tricritical point, i.e. a change in the order of phase transition. This tendency manifests itself more clearly for the PBaGO solid solutions (see Table 2). In addition, one should remind specific behaviour of the optical activity in the PBaGO solid solutions, which is improper for the second-order phase transitions. All of these facts suggest that a hypothetical tricritical point could exist on the $x, T$-phase diagram, at least for the PBaGO solid solutions. However, the concentration behaviour of the $\beta$ coefficient for the PBaGO solid solutions obtained in the present work is more pronounced than that obtained in [7] on the basis of dielectric measurement. This fact might be associated with some difference between the temperature dependences of optical activity and spontaneous polarization for the solid solutions under study.

Table 1. Coefficients of thermodynamic potential for the PGSO solid solutions.

\begin{tabular}{|c|c|c|c|c|}
\hline $\mathrm{x}$ & $\left(\gamma_{33}^{-1}\right)_{T<T_{c}} /\left(\gamma_{33}^{-1}\right)_{T>T_{c}}$ & $\mathrm{C}, 10^{4} \mathrm{~K}$ & $\alpha, 10^{-4} \mathrm{~K}^{-1}$ & $\beta, \mathrm{m}^{4} / \mathrm{C}^{2}$ \\
\hline 0 & 2.9 & 1.0 & 0.5 & 7.5 \\
& $\begin{array}{c}3[1] \\
2.4 \text { for } \mathrm{Cr}^{3+} \text { doped } \\
\text { PGO [13] }\end{array}$ & $\begin{array}{c}1.04[1] \\
0.4 \pm 0.1 \text { for } \mathrm{Cr}^{3+} \\
\text { doped PGO [13] }\end{array}$ & $\begin{array}{c}0.48[1] \\
1.25 \text { for } \mathrm{Cr}^{3+} \\
\text { doped PGO [13] }\end{array}$ & $\begin{array}{c}16.55[1] \\
5{\text { for } \mathrm{Cr}^{3+} \text { doped }}^{\mathrm{PGO}[13]}\end{array}$ \\
\hline 0.1 & 3.3 & 1.1 & 0.45 & 5.6 \\
\hline 0.4 & 4.0 & 1.4 & 0.36 & 0.9 \\
\hline
\end{tabular}

Table 2. Coefficients of thermodynamic potential for the PBaGO solid solutions.

\begin{tabular}{|c|c|c|c|c|}
\hline$x$ & $\left(\gamma_{33}^{-1}\right)_{T<T_{c}} /\left(\gamma_{33}^{-1}\right)_{T>T_{c}}$ & $\mathrm{C}, 10^{4} \mathrm{~K}$ & $\alpha, 10^{-4} \mathrm{~K}^{-1}$ & $\beta, \mathrm{m}^{4} / \mathrm{C}^{2}$ \\
\hline 0 & 2.9 & 1.0 & 0.5 & 7.5 \\
& $2[7]$ & $1.32[7]$ & $0.38[7]$ & $2.8 \times 10^{-10}(\mathrm{CGS} \text { units })^{-2}[7]$ \\
\hline 0.02 & 4.82 & 3.22 & 0.16 & 0.45 \\
& $1.7[7]$ & $1.5[7]$ & $0.33[7]$ & $2.5 \times 10^{-10}(\mathrm{CGS} \text { units })^{-2}[7]$ \\
\hline 0.05 & 1.50 & 6.6 & 0.08 & 0.1 \\
& $1.6[7]$ & $1.75[7]$ & $0.29[7]$ & $2.1 \times 10^{-10}(\mathrm{CGS} \text { units })^{-2}[7]$ \\
\hline
\end{tabular}

It is clearly seen from Fig. 9 that the recalculated coefficient $\tilde{\gamma}_{333} \simeq \frac{\gamma_{333}}{\varepsilon_{0} \varepsilon_{33}}$ depends on neither temperature nor concentration of the substitution ions. Hence, one can conclude that the difference of the magnitude of electrogyration effect observed for the solid solutions of PGSO and PBaGO is caused solely by the critical behaviour of dielectric permittivity in the vicinity of the phase transition temperature. 


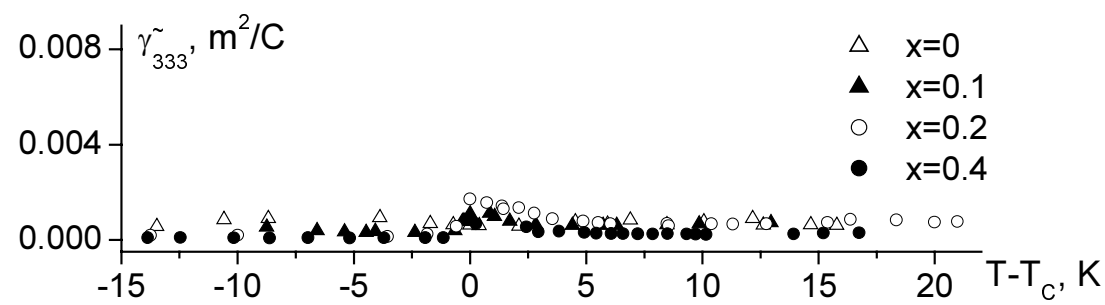

(a)

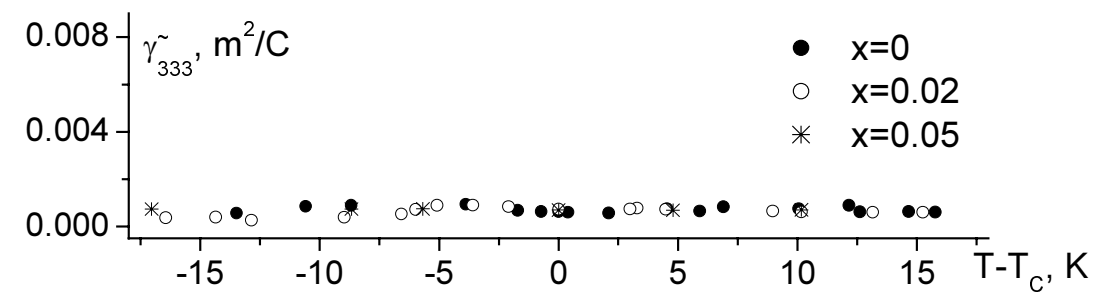

(b)

Fig. 9. Temperature dependences of $\tilde{\gamma}_{333}$ coefficient for the PGSO (a) and PBaGO (b) solid solutions.

\section{Conclusion}

We conclude that the substitutions of $\mathrm{Pb}$ with $\mathrm{Ba}$ and $\mathrm{Ge}$ with $\mathrm{Si}$ do not influence on the magnitude of electrogyration coefficient defined in the units of reciprocal electric polarization. The difference of electrogyration coefficients represented in the units of reciprocal electric field strength observed in the vicinity of phase transition temperature is determined by critical behaviour of the dielectric permittivity at $T_{c}$. For both the PGSO and PBaGO solid solutions, the substitutions lead to decrease in the Curie temperature and decrease in the coefficients of thermodynamic potential. Small-percentage substitutions of $\mathrm{Pb}$ with $\mathrm{Ba}$ in the $\mathrm{PBaGO}$ solid solutions imposes a situation when both the thermodynamic coefficients tend simultaneous to zero, thus suggesting existence of a hypothetical tricritical point on the $x, T$-phase diagram. We have also revealed a measurable cubic electrogyration effect in the solid solutions of PGSO and have determined the corresponding parameter.

\section{References}

1. Iwasaki H, Miyazawa S, Koizumi H, Sugii K, and Niizeki N, 1972. Ferroelectric and optical properties of $\mathrm{Pb}_{5} \mathrm{Ge}_{3} \mathrm{O}_{11}$ and its isomorphous compound $\mathrm{Pb}_{5} \mathrm{Ge}_{2} \mathrm{SiO}_{11}$. J. Appl. Phys. 43: 4907-4915.

2. Novik VK and Gavrilova ND, 2000. Low-temperature pyroelectricity. Fiz. Tverd. Tela 42: 991-1008.

3. Aizu K, 1964. Reversal in optical rotatory power - "gyroelectric" crystals and "hypergyroelectric" crystals. Phys. Rev. 133: A1584-A1588 
4. Newnham R E and Cross L E, 1974. Ambidextrous crystals. Endeavour. 13 18-22.

5. Vlokh OG, Lazko LA and Shopa YI, 1981. Electrooptic and Electrogyration Properties of the Solid Solutions on the Basis of Lead Germanate. Phys. Stat. Sol. (a). 65: $371-378$.

6. Vlokh OG, Lazko LA and ShopaYI, 1980. Electrogyration and Electro-Optic Properties of the Solid Solutions on the Basis of Lead Germanate. J. Phys. Soc. Jap. Suppl. B. 49: 150-151.

7. Strukov BA, Sinyakov EV, Mayshchik EP, Minaeva KA, Monya VG and Vlokh OG, 1977. Ultrasonic relaxation, smearing of phase transition and some physical properties of lead germanate crystals and solid solutions on their basis. Izv. AN USSR, Ser. Fiz. 41: 692-699.

8. Vlokh OG, Sinyakov EV, Lazko LA, Monya VG and Klimov IM, 1977. Electrogyration and dielectric properties of the solid solutions on the basis of lead germanate. Fiz. Tverd. Tela 19: 149-152.

9. Vlokh OG, Zheludev IS and Sergatyuk VA, 1984. Electric and magnetic gyration in crystals. Izv. AN SSSR, Ser. Fiz. 48: 1771-1776.

10. Vlokh O and Sergatyuk V, 1987. Magneto-polarization optical activity in crystals of lead germanates type. Izv. AN SSSR, Ser. Fiz. 51: 2183-2186.

11. Pryadko LD, Gnatovsky O, Semenets TI and Linnik VG, 1996. Study of the photorefractive effect in crystals $\mathrm{Pb}_{5} \mathrm{Ge}_{3} \mathrm{O}_{11}: \mathrm{Cu}$ and $\mathrm{Pb}_{5} \mathrm{Ge}_{3} \mathrm{O}_{11}$. Proc. SPIE. 2795: 208-211.

12. Pryadko LD, Gnatovsky O, Linnik VG and Yanchook Z, 1998. Photorefractive effect in $\mathrm{Pb}_{5} \mathrm{Ge}_{3} \mathrm{O}_{11}: \mathrm{Nd}$ crystals. Proc. SPIE. 3488: 259-262.

13. Adamenko D, Klymiv I, Duda VM, Vlokh R and Vlokh O, 2007. Electrically and magnetically induced optical rotation in $\mathrm{Pb}_{5} \mathrm{Ge}_{3} \mathrm{O}_{11}$ : $\mathrm{Cr}$ crystals at the phase transition. Electrogyration effect in $\mathrm{Pb}_{5} \mathrm{Ge}_{3} \mathrm{O}_{11}$ :Cr. Ukr. J. Phys. Opt. 8: 42-53.

14. Goto Y and Sawaguchi E, 1979. Electric conductivity of ferroelectric $\mathrm{Pb}_{5} \mathrm{Ge}_{3} \mathrm{O}_{11} \mathrm{~J}$. Phys. Soc. Jap. 46: 1580-1582.

15. Vlokh OG, Sinyakov EV, Lazko LA, Shopa YaI and Kreycherek AYa, 1980. Electrogyration and dielectric properties of the lead germanate crystals with silicon admixture. Fiz. Tverd. Tela 22: 227-229.

16. Shaskolskaya MP Acoustic crystals. Moscow: Nauka (1982).

17. Smolenskiy GA Physics of ferroelectric phenomena. Leningrad: Nauka (1985). 